\title{
ON STRONG SUMMABILITY OF A FOURIER SERIES
}

FU TRAING WANG

Let $s_{n}(x)=a / 2 \sum_{\nu=1}\left(a_{\nu} \cos \nu x+b_{\nu} \sin \nu x\right)$ be the partial sum of the Fourier series of an integrable periodic function $f(t)$ of period $2 \pi$, and let $\phi(t)=\{f(x+t)+f(x-t)-2 s\} / 2$. We shall establish the following result (Hardy-Littlewood [1] $]^{1}$ ).

THEOREM. If

$$
\int_{0}^{t}|\phi(u)|\left\{1+\log ^{+}|\phi(u)|\right\} d u=o(t), \quad \text { as } t \rightarrow 0,
$$

then $\sum_{p=0}^{n}\left|s_{\nu}(x)-s\right|^{2}=o(n \log \log n)$, as $n \rightarrow \infty$.

To prove this theorem we require the following lemmas.

LEMMA 1. If

$$
\int_{0}^{t}|\phi(u)| d u=o(t), \quad \text { as } t \rightarrow 0,
$$

then

$$
\sum_{\nu=0}^{n}\left|s_{\nu}(x)-s\right|^{2}=\frac{1}{\pi^{2}} \int_{1 / n}^{\delta} \frac{\phi(t)}{t^{2}} d t \int_{1 / n}^{t} \phi(u) \frac{\sin n(u-t)}{u-t} d u+o(n) .
$$

Proof. By (2), for $\nu \leqq n$,

$$
s_{\nu}(x)-s=\frac{2}{\pi} \int_{1 / n}^{\delta} \phi(t) \frac{\sin \nu t}{t} d t+o(1) .
$$

Hence

$$
\begin{aligned}
\sum_{\nu=0}^{n}\left|s_{\nu}(x)-s\right|^{2}= & \frac{4}{\pi^{2}} \int_{1 / n}^{\delta} \int_{1 / n}^{\delta} \frac{\phi(u) \phi(t)}{u t}\left\{\sum_{\nu=1}^{n} \sin v t \sin v u\right\} d u d t+o(n) \\
= & \frac{2}{\pi^{2}} \int_{1 / n}^{\delta} \int_{1 / n}^{\delta} \frac{\phi(u) \phi(t)}{u t} \frac{\sin n(u-t)}{u-t} d u d t \\
& +\frac{2}{\pi^{2}} \int_{1 / n}^{\delta} \int_{1 / n}^{\delta} \frac{\phi(u) \phi(t)}{u t} \frac{\sin n(u+t)}{u+t} d u d t+o(n) \\
= & J_{1}+J_{2}+o(n) .
\end{aligned}
$$

Received by the editors December 15, 1943.

1 Numbers in brackets refer to the references listed at the end of the paper. 
Set $\Phi(t)=\int_{0}^{t}|\phi(u)| d u$. Then

But

$$
J_{2}=O\left\{\int_{1 / n}^{\delta} \frac{|\phi(t)|}{t^{2}} d t \int_{1 / n}^{t} \frac{|\phi(u)|}{u} d u\right\} \text {. }
$$

$$
\int_{1 / n}^{\delta} \frac{|\phi(t)|}{t^{2}} d t \int_{1 / n}^{\delta} \frac{|\phi(u)|}{u} d u=O\left\{\int_{1 / n}^{\delta} \frac{|\phi(t)|}{t^{2}} \log n t d t\right\},
$$

and

$$
\begin{aligned}
\int_{1 / n}^{\delta} \frac{|\phi(t)|}{t^{2}} \log n t d t= & {\left[\frac{\Phi(t)}{t^{2}} \log n t\right]_{1 / n}^{\delta}+2 \int_{1 / n}^{\delta} \frac{\Phi(t)}{t^{8}} \log n t d t } \\
& -\int_{1 / n}^{\delta} \frac{\Phi(t)}{t^{3}} d t=o(n)+o\left(n \int_{1}^{\delta n} \frac{\log t}{t^{2}} d t\right) \\
& +o\left(\int_{1 / n}^{\delta} \frac{d t}{t^{2}} d t\right)=o(n) .
\end{aligned}
$$

Hence

$$
J_{2}=o(n) \text {. }
$$

By inversion of the order of integration, and the resolution of $1 / t(t-u)$ into partial fractions, we get

$$
\begin{aligned}
J_{1}= & \frac{2}{\pi^{2}} \int_{1 / n}^{\delta} \frac{\phi(t)}{t^{2}} d t \int_{1 / n}^{t} \phi(u) \frac{\sin n(n-t)}{u-t} d u \\
& +o\left(\int_{1 / n}^{\delta} \frac{|\phi(t)|}{t^{2}} d t \int_{1 / n}^{t} \frac{|\phi(u)|}{u} d u\right. \\
= & \frac{1}{\pi^{2}} \int_{1 / n}^{\delta} \frac{\phi(t)}{t^{2}} d t \int_{1 / n}^{t} \phi(u) \frac{\sin n(u-t)}{u-t} d u+o(n) .
\end{aligned}
$$

Lemma 1 follows from (4) and (5).

LEMMA 2. If $P(u)$ and $F(u)$ are non-negative integrable functions in $(a, b)$, and $\psi(x)$ is a convex function in $(0, \infty)$,

$$
\begin{aligned}
\psi\left\{\int_{a}^{b} P(u) F(u) d u / \int_{a}^{b} P(u) d u\right\} & \\
& \leqq \int_{a}^{b} P(u) \psi\{F(u)\} d u / \int_{a}^{b} P(u) d u .
\end{aligned}
$$

This is the well known Jensen inequality (Hardy-LittlewoodPólya [2]). 
Proof of The theorem. Since $1+\log ^{+}|\phi(u)| \geqq 1$,

$$
\int_{0}^{t}|\phi(u)| d u \leqq \int_{0}^{t}|\phi(u)|\left\{1+\log ^{+}|\phi(u)|\right\} d u=o(t) .
$$

Hence by Lemma 1 it suffices to prove that

$$
G_{n}=\int_{1 / n}^{\delta} \frac{\phi(t)}{t^{2}} d t \int_{1 / n}^{t} \phi(u) \frac{\sin n(u-t)}{u-t} d u=o(n \log \log n) .
$$

Now

(6)

$$
\begin{aligned}
G_{n}= & \int_{1 / n}^{n^{-1} \log n} \frac{\phi(t)}{t^{2}} d t \int_{1 / n}^{t} \phi(u) \frac{\sin n(u-t)}{u-t} d u \\
& +\int_{n^{-1} \log n}^{\delta} \frac{\phi(t)}{t^{2}} d t \int_{1 / n}^{t-n^{-1} \log n} \phi(u) \frac{\sin n(u-t)}{u-t} d u \\
& +\int_{n^{-1} \log n}^{\delta} \frac{\phi(t)}{t^{2}} d t \int_{t-n^{-1} \log n}^{t} \phi(u) \frac{\sin n(u-t)}{u-t} d u \\
= & I_{1}+I_{2}+I_{3} .
\end{aligned}
$$

By (2) we have

$$
I_{1}=\int_{1 / n}^{n^{-1} \log n} \frac{|\phi(t)|}{t^{2}} O(n t) d t=o(n \log \log n),
$$

and

$$
I_{2}=\int_{n^{-1} \log n}^{\delta} \frac{|\phi(t)|}{t^{2}} O\left(\frac{n}{\log n} t\right) d t=o(n) .
$$

Now set

and

$$
\begin{aligned}
P(u) & =\left|\frac{\sin n(u-t)}{u-t}\right|, \\
k & =\int_{t-n^{-1} \log n}^{t} P(u) d u \sim \log \log n,
\end{aligned}
$$

$$
J_{n}(t)=\int_{t-n^{-1} \log n}^{t}|\phi(u)| P(u) d u .
$$

Applying Lemma 2 with $\psi(x)=x\left(1+\log ^{+} x\right)$, we find

$$
\psi\left\{\frac{J_{n}(t)}{k}\right\} \leqq \frac{\int_{t-n^{-1}}^{t} \log n|\phi(u)|\left\{1+\log ^{+}|\phi(u)| P(u) d u\right.}{k} \leqq \frac{n t}{k} .
$$


Hence

$$
J_{n}(t) \leqq n t\left\{1+\log +\frac{J_{n}(t)}{k}\right\}^{-1}
$$

If we suppose now that

$$
J_{n}(t) \geqq A_{1} n t / \log n t k^{-1},
$$

we find $1+\log ^{+}\left(J_{n}(t) / k\right) \geqq A \log (n t / k)$, for $n t / k \geqq \log n / \log \log n$. Hence

$$
J_{n}(t) \leqq A_{2} n t / \log n t k^{-1},
$$

for all values of $n$ and $t$ such that (9) and the relation $n t \geqq \log n$ hold true.

If (9) does not hold,

$$
J_{n}(t) \leqq A_{1} n t / \log n t k^{-1} .
$$

By (10) and (11) we have then

$$
J_{n}(t) \leqq A_{3} n t / \log n t k^{-1}
$$

for all values of $n$ and $t$ for which $n t \geqq \log n$.

By (12)

$$
\begin{aligned}
\left|I_{3}\right| \leqq & \int_{n^{-1} \log n}^{\delta} \frac{|\phi(t)|}{t^{2}} J_{n}(t) d t \leqq A \int_{n^{-1} \log n}^{\delta} \frac{|\phi(t)|}{t^{2}} \frac{n}{\log (n / \log \log n)} d t \\
\leqq & A n\left\{\left[\frac{\Phi(t)}{t} \frac{1}{\log (n t / \log \log n)}\right]_{n^{-1} \log n}^{\delta}\right. \\
& +\int_{n^{-1} \log n}^{\delta} \frac{\Phi(t)}{t^{2}} \frac{d t}{\log (n t / \log \log n)} \\
& \left.+\int_{n^{-1} \log n}^{\delta} \frac{\Phi(t)}{t^{2}} \frac{d t}{(\log (n t / \log \log n))^{2}}\right\} \\
= & o(n \log \log n) .
\end{aligned}
$$

From this inequality and (6), (7), and (8) we draw

$$
G_{n}=o(n \log \log n) \text {. }
$$

In conclusion I remark that by the above method I established in a recent paper the following result, which is a generalization of Hardy and Littlewood's theorem [1].

If

$$
\int_{0}^{t}|\phi(u)| d u=O(t) \text { and } \quad \int_{0}^{t} \phi(u) d u=o(t) \quad \text { as } t \rightarrow 0 \text {, }
$$


then

$$
\begin{gathered}
\sum_{\nu=0}^{n}\left|s_{\nu}(x)-s\right|^{2}=o(n \log n), \quad \text { as } n \rightarrow \infty . \\
\text { REFERENCES }
\end{gathered}
$$

1. G. H. Hardy and J. E. Littlewood, On strong summability for a Fourier series, Fund. Math. vol. 25 (1935).

2. G. H. Hardy, J. E. Littlewood, and G. Pólya, Inequalities, Cambridge, 1934, p. 151.

National University of Chekiang 